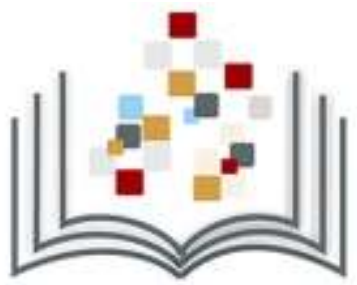

\title{
ORBITAL - THE ELECTRONIC JOURNAL OF CHEMISTRY: HISTÓRICO DA CRIAÇÃO E ANÁLISE DE PERFIL
}

\author{
Adilson Beatriz $^{1 *}$, Grégoire Jean-François Demets ${ }^{2}$ \\ 1 Universidade Federal de Mato Grosso do Sul - Instituto de Química - Campo Grande \\ (MS) - Brasil \\ 2Universidade de São Paulo - Faculdade de Filosofia, Ciências e Letras de Ribeirão \\ Preto - Ribeirão Preto (SP) - Brasil \\ *Autor correspondente: adilson.beatriz@ufms.br
}

\section{Como citar:}

BEATRIZ, Adilson; DEMETS, Grégoire Jean-François. Orbital - the electronic journal of chemistry: histórico da criação e análise de perfil. In: WORKSHOP DE EDITORAÇÃO CIENTÍFICA, 9., 2016, São Paulo. Anais... São Paulo: Associação Brasileira de Editores Científicos, 2016. p. 33-38

DOI: http://dx.doi.org/10.21452/wec.IXwec.2016.0007

\section{RESUMO}

A revista científica Orbital: The Electronic Journal of Chemistry foi criada por professores da Universidade Federal de Mato Grosso do Sul (UFMS) em 2008, visando a publicação de artigos originais em todas as áreas da química e suas interfaces com a farmácia, biologia e física. A missão da Orbital é de ser uma revista científica de química, totalmente eletrônica e com acesso livre e irrestrito e sem nenhuma taxa, tanto para o autor como para o leitor. A revista tem periodicidade trimestral e publica edições temáticas também. Os artigos são submetidos a processo de revisão por pares. Teve seu primeiro número lançado em 2009. Desde 2015 possui atribuições do DOI e está indexada em diversas bases de dados, incluindo ESCI (Web of Science), DOAJ, CAS, EBSCO, Google Scholar, Latindex, dentre outros. Neste trabalho, estamos apresentando um histórico do processo de criação e também uma análise do perfil da revista do período de 2009 a 2016.

\section{INTRODUÇÃO}

\begin{abstract}
"O periódico científico é o principal canal formal de disseminação da ciência, por meio do qual os pesquisadores tornam públicas as suas pesquisas. A publicação de artigos originais resultantes de pesquisa científica constitui a primeira informação que inicia o diálogo científico. Ao publicar, o periódico científico fornece elementos para a recuperação da informação por meio dos títulos, sumários, abstracts ou resumos que, por meio de um padrão necessário, permitem o acesso e a recuperação de artigos e autores, e dos próprios títulos de periódicos, pelo processo de tratamento, sistemas de indexação de bibliotecas e bases de dados que, por sua vez, vão permitir a execução de outros serviços de informação, como análise de citação, fator de impacto, entre outros" (VALÉRIO, 2015).
\end{abstract}

Na era da informática, através da internet, todo esse processo se tornou mais fácil, ágil e disponível para toda a comunidade. Nesse sentido, o Sistema Eletrônico de Editoração de Revistas (SEER) foi desenvolvido para a construção e gestão de uma 
publicação periódica eletrônica. Esta ferramenta contempla ações essenciais à automação das atividades de editoração de periódicos científicos. O SEER foi traduzido e customizado pelo Instituto Brasileiro de Informação em Ciência e Tecnologia (Ibict) baseado no software desenvolvido pelo Public Knowledge Project (PKP) o Open Journal Systems (OJS) da University of British Columbia (UBS) ${ }^{1}$.

Em 2008, um grupo de professores da Universidade Federal de Mato Grosso do Sul (UFMS) pensou na criação de uma revista científica de química, pois, se comparado a países desenvolvidos, ainda era muito pequeno o número de revistas voltadas para esta ciência, editadas no Brasil. Nesse sentido, um periódico eletrônico seria certamente bem-vindo e viria a preencher uma lacuna existente na edição de revistas eletrônicas na área de química. Nessa direção, foi criada a revista Orbital: Revista Eletrônica de Química/The Electronic Journal of Chemistry, com a missão de ser uma revista científica totalmente eletrônica e com acesso livre e irrestrito e sem nenhuma taxa, tanto para o autor como para o leitor. Com a criação da revista Orbital, a primeira revista eletrônica de química do estado de Mato Grosso do Sul, a UFMS se alçaria à posição de vanguarda na produção e disseminação do conhecimento científico dessa área.

A publicação de artigos na área de química é muito competitiva e, além disso, os órgãos brasileiros de fomentos à pesquisa dão grande importância a publicações feitas em revistas que tenham fator de impacto alto e que estejam classificados pela Qualis Capes. Dessa forma, a captação de artigos de cientistas brasileiros seria um problema para uma revista recém-criada, tendo como editores pesquisadores de renome apenas regional e sem treinamento para esta função vital. No entanto, esse desafio inicial foi superado e a revista Orbital em breve celebrará o seu oitavo aniversário de publicação ininterrupta, o que para uma revista científica completamente livre de encargos para autores e leitores é algo que achamos muito gratificante e desafiador. Ao longo dos anos, a revista teve como objetivo publicar artigos em todas as áreas da química, aceitando contribuições em três idiomas: inglês, português e espanhol. Neste trabalho, estamos apresentando um histórico do processo de criação e também uma análise do perfil da revista do período de 2009 (primeiro número) até a presente data.

\section{METODOLOGIA}

Foi realizado um estudo descritivo retrospectivo no período de 2009 a 2016 . Foram avaliados na plataforma SEER da revista Orbital $^{2}$ os dados referentes ao número médio de artigos por edição (de acordo com a modalidade), número médio de autores por artigo, porcentagem das contribuições nas línguas aceitas, contribuição de estrangeiros por país e contribuição dos brasileiros por unidade da federação. A análise descritiva foi realizada utilizando-se números absolutos, percentagens e médias.

\section{RESULTADOS E DISCUSSÃO}

Histórico da criação: a criação da revista foi aprovada pelo Conselho Editorial da Fundação Universidade Federal de Mato Grosso do Sul, através da resolução no 1 , de 14 de janeiro de 2008, tendo como editores-chefes os professores Adilson Beatriz e Marcos Serrou do Amaral e editores associados os professores Dênis Pires de Lima e Giuseppe A. Câmara, todos da UFMS. A revista foi concebida para aceitar manuscritos escritos em português, espanhol ou inglês referente a todas as áreas da química e suas interfaces com a farmácia, biologia e física. A contribuição poderia se encaixar dentro de uma das modalidades a seguir: artigos originais completos, artigos de revisão, artigos sobre educação, história da química, comunicação breve, nota técnica e carta ao editor. A periodicidade trimestral foi escolhida com vista a atender as recomendações da Scientific Electronic Library Online (SciELO), para futuro pedido de indexação. 
Também visando atender os critérios das bases de dados mais importantes, o Conselho Editorial foi estruturado necessariamente multi-institucional, constituído por pesquisadores especialistas distribuídos uniformemente, tanto científica como geograficamente. Portanto, o Conselho Editorial foi inicialmente constituído por pesquisadores doutores da UFMS e de outras instituições brasileiras, incluindo os estados de São Paulo (SP), Mato Grosso (MT), Espírito Santo (ES), Pernambuco (PE), Sergipe (SE), Paraíba (PB), Bahia (BA), Paraná (PR) e do próprio estado de Mato Grosso do Sul (MS). Vale ressaltar que todos os pesquisadores aceitaram prontamente ao convite para pertencerem ao corpo editorial da revista, demonstrando, assim, apoio total à ideia de criação de uma revista eletrônica de química. Nesse mesmo ano, iniciamos o processo de divulgação do periódico através de listas de e-mails e divulgação em diversas universidades do Brasil e do exterior, incluindo chamada para submissão de artigos no Boletim Eletrônico da Sociedade Brasileira de Química. Além disso, começamos o processo de indexação em diversas bases de dados e repositórios digitais, aumentando a visibilidade da revista, permitindo que pudéssemos lançar o primeiro número com 9 contribuições (7 artigos completos e 2 artigos de revisão), 5 desses na língua inglesa e 4 em português. Cinco contribuições foram de autores indianos e os outros de autores brasileiros dos estados de MS, RS e SP. Desde então, as capas virtuais de cada número são elaboradas voluntariamente pelo Prof. Álvaro Takeo Omori da Universidade Federal do ABC.

Em abril de 2009, a revista foi adicionada no Directory of Open Access Journals (DOAJ), naturalmente a base de dados mais importante de revistas de acesso aberto de todo o mundo. A partir desse diretório, o periódico foi incorporado em outras bases de dados, incluindo bibliotecas digitais das maiores universidades internacionais. Em 2010, a Orbital foi indexada no CAS Source Index (CASSI) Search Tool, que permite pesquisadores encontrarem os artigos publicados usando a base de dados da SCIFINDER, uma poderosa ferramenta destinada a pesquisas na área de química e afins. Um fato marcante aconteceu em dezembro de 2012, quando a revista Orbital foi classificada com Qualis B5 pela coordenação da área de química da Capes. Nas áreas de engenharia I e interdisciplinar a revista recebeu a classificação B3; B4 em engenharias II e B5 nas áreas de biotecnologia, materiais e farmácia. As áreas de astronomia/física e ciências agrárias I atribuíram Qualis C. Nas avaliações subsequentes (2013 e 2014), a revista obteve as seguintes classificações: B5: química, materiais e biotecnologia. B4: ensino, ciências ambientais, engenharias II e III. B3: engenharias I. C: astronomia/física, farmácia e zootecnia/recursos pesqueiros. As publicações são incluídas a partir dos relatórios dos Programas de Pós-Graduação de cada área (Coleta Capes). Este importante fato demonstrou que a revista Orbital estava progressivamente conquistando o reconhecimento da comunidade científica brasileira. A classificação pela área de química da Capes foi de extrema importância, uma vez que este sistema avalia a geração de conhecimento nas universidades brasileiras e a maneira que este conhecimento é difundido na sociedade. Uma das recomendações da área de química da Capes, para permanecer com webqualis B5, foi a necessidade de indexação da revista em banco de dados como Scopus, Web of Science ou SciELO, o que permite a medição do fator de impacto. Levando em consideração essas recomendações, era imperativo a internacionalização do seu Conselho Editorial, o que foi feito através de convites a pesquisadores de diversas partes do mundo. Atualmente, os membros do Conselho Consultivo Internacional da Revista Orbital são de países como Egito, Turquia, Canadá, China, Espanha, Portugal, Irã, África do Sul e Argentina. A partir de 2015, a Orbital passa a apresentar o Digital Object Identifier (DOI), dando um salto para a visibilidade dos artigos publicados. Em 2016, a revista ganhou a contribuição de 4 editores associados internacionais (provenientes de universidades do Japão, Irlanda, França e Austrália) e, nesse mesmo ano, a Orbital passou a aceitar apenas artigos escritos em inglês. Essas ações culminaram na inclusão da revista Orbital na Emerging Sources Citation Index (ESCI), uma nova edição do Web of Science, em setembro de 2016. As revistas deste índice são consideradas pela Thomson Reuters para serem aceitas no Expanded Science Citation Index (SCIE). A inclusão da Orbital no ESCI demonstra a dedicação dos editores em oferecer o conteúdo 
científico mais relevante e influente para os seus leitores.

\section{ANÁLISE DE PERFIL DA REVISTA (ORBITAL EM NÚMEROS)}

Número de artigos por edição: Com exceção de cartas ao editor, que até o momento não teve nenhuma submissão, foram aferidos os números médios de contribuições nas modalidades: artigos originais completos, artigos de revisão, artigos sobre educação, história da química, comunicação breve e notas técnicas. Como podemos observar na figura 1, o número médio de artigos por edição vem aumentando gradativamente, mas ainda há um evidente descompasso entre a publicação de artigos originais completos e as outras modalidades. Podemos considerar que a área de educação em química tem muito a crescer e necessita de atenção especial na divulgação da revista para pesquisadores que atuam nesse tema. Vale lembrar que a área de ensino em química classificou a revista como B4 no Qualis Capes.

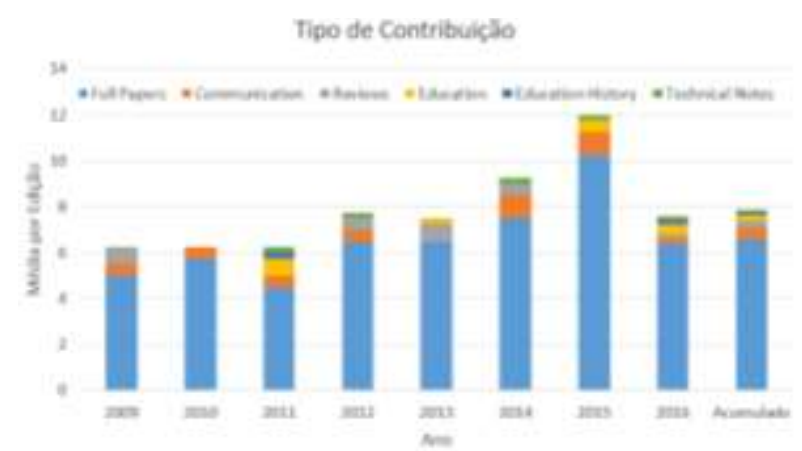

Figura 1: Número médio de contribuições nas modalidades aceitas pela revista.

Número médio de autores por artigo: a figura 2 mostra o número médio de autores por artigo, que indubitavelmente vem aumentando sutilmente. Artigos com mais de 5 autores são frequentemente de autores brasileiros. Seria recomendável a exigência de uma declaração sobre a contribuição de cada autor no artigo, como já fazem muitas revistas importantes da área.

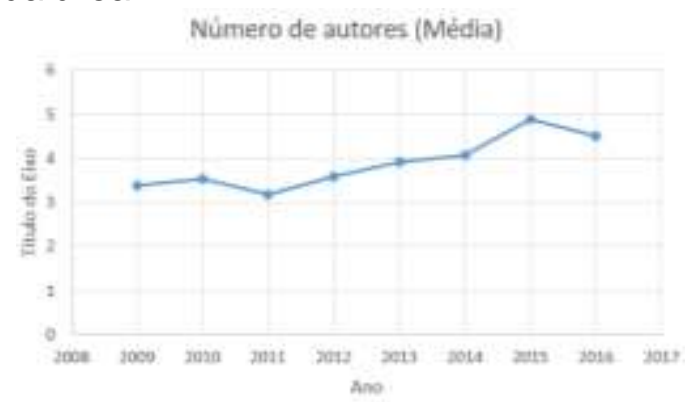

Figura 2: Número médio de autores por artigo, período de 2009-2016.

Idioma: a figura 3 apresenta a proporção entre inglês, português e espanhol. Observa-se que o espanhol, contribui minimamente. Em 100\% dos casos, as submissões em espanhol, não são de autores hispanófonos. As contribuições em inglês são mais frequentes em todos os anos analisados. Os dados de 2016 são ainda parciais, podendo mudar com o fechamento do último número desse ano. A média acumulada no período analisado foi de $64,24 \%$ para inglês, $34,04 \%$ para português e $1,99 \%$ para espanhol. 


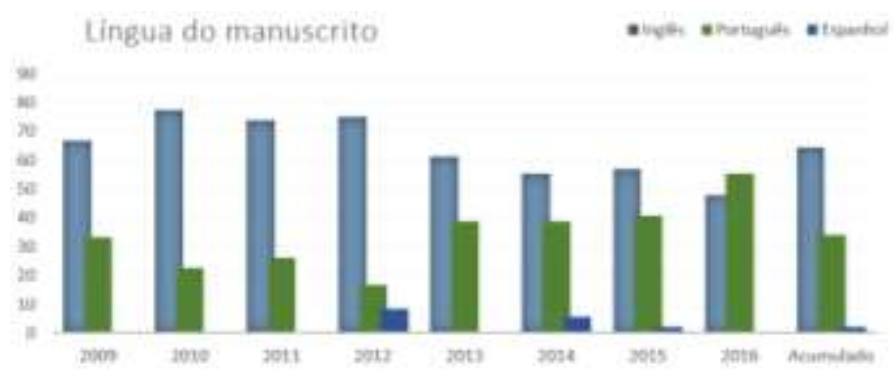

Figura 3: Proporção entre as línguas inglesa, portuguesa e espanhola nos manuscritos aceitos no período de 2009-2016.

Contribuição de estrangeiros: a figura 4 apresenta a percentagem de artigos publicados por autores estrangeiros. Podemos observar que de 2009 a 2012, a contribuição internacional era maioria. A partir de 2013, houve uma sensível queda no número de contribuições estrangeiras, atestando uma maior penetração da Orbital no cenário nacional, o que pode indicar que a classificação do periódico no Qualis Capes fez aumentar o interesse de autores brasileiros em publicar na revista.

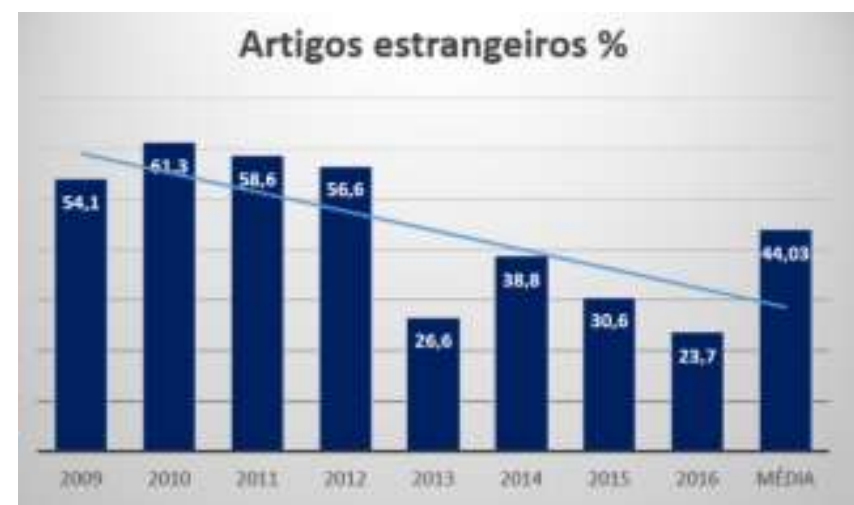

Figura 4: Percentual de contribuições de estrangeiros.

Contribuição de estrangeiros por país: outro dado analisado foi a contribuição de estrangeiros por país, apresentado na figura 5 . A Índia ainda é o país que mais aparece nas edições da Orbital (média acumulada de 59,5\%), o que pode indicar a necessidade de melhorar a divulgação da revista na América Latina, Europa e países como China, Estados Unidos e Rússia. Edições temáticas e publicação de artigos de revisão por autores convidados seriam boas estratégias para atrair estes pesquisadores.

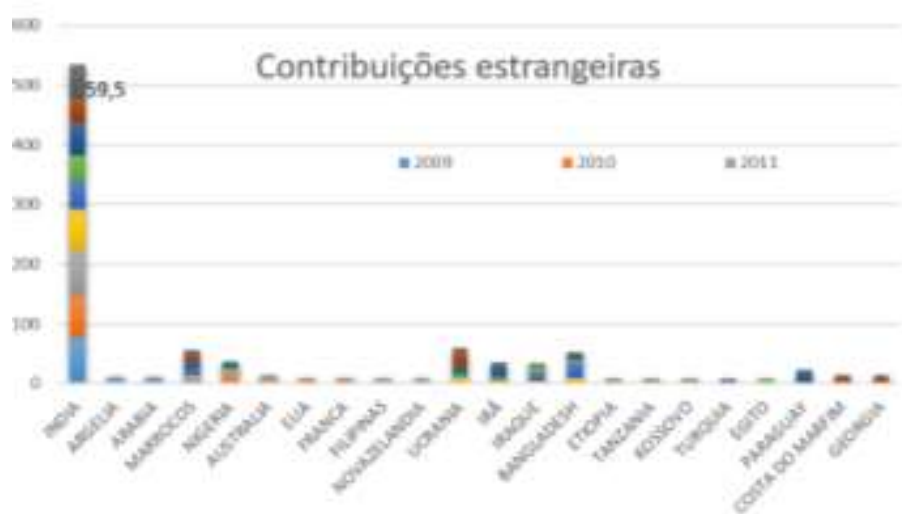

Figura 5: Contribuição de estrangeiros por país.

Contribuição por unidade da federação: Recebemos contribuições de 21 estados brasileiros. O estado de SP se destaca com a média acumulada de $22,73 \%$. Há também o destaque natural para MS (21\%) e o PR, aparecendo com $12,99 \%$ das contribuições no período analisado. Outros estados do Sul, Sudeste e alguns do Nordeste também aparecem com frequência. Podemos observar que ainda há desequilíbrio entre as 
regiões da Federação (figura 6).

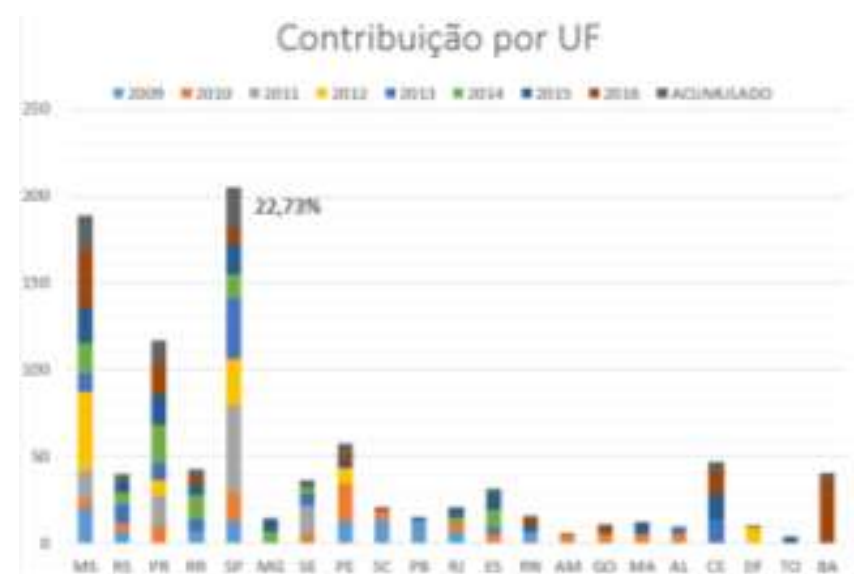

Figura 6: Contribuições de autores brasileiros por Unidade da Federação.

\section{CONCLUSÕES}

A partir do perfil numérico discutido torna-se visível a importância da indexação da revista a bases de dados como Scopus, Web of Science e Scielo, visando a obtenção de índices para maior visibilidade e assim obter o tão almejado fator de impacto e a inserção internacional definitiva do periódico. Para que isso aconteça, a revista precisa ter recursos para assegurar um mínimo de profissionalismo no processo de edição dos artigos aceitos, bem como disponibilizar normas claras e acessíveis aos autores. A rapidez no trâmite editorial é de fundamental importância para atrair artigos de qualidade de todas as partes de mundo.

\section{REFERÊNCIAS}

VALÉRIO, P. M. O periódico científico. In: Curso de Atualização sobre Avaliação do Trabalho Científico. Petrópolis: ABEC, 2005. Disponível em: Disponível em: $<$ http://www.Incc.br/abec/eventos.php>. Acesso em: 15 set. 2007.

PALAVRAS-CHAVE: Periódico. Revista científica. Química. Mato Grosso do Sul. 